\title{
Multilayer Photonic Crystal for Spectral Narrowing of Emission
}

\author{
Zhanfang LIU ${ }^{1,2}$, Dan WANG ${ }^{2}$, Jiao FENG $^{3}$, Hong ZHOU ${ }^{1}$, Guannan ZHANG ${ }^{1}$, \\ Zhenwen SUN ${ }^{1}$, Jun ZHU $^{1}$, Kai SONG ${ }^{2}$ * \\ ${ }^{1}$ Institute of Forensic Science, Ministry of Public Security, Beijing, 100038, P.R.China \\ ${ }^{2}$ CAS Key Laboratory of Bio-inspired Materials and Interfacial Science, Technical Institute of Physics and Chemistry, \\ Chinese Academy of Sciences, Beijing 100190, China \\ ${ }^{3}$ Key Laboratory of Science and Technology on High Energy Laser, Institute of Chemical Materials and Advanced \\ Materials Center, China Academy of Engineering Physics, Mianyang, 621900, China
}

crossref http://dx.doi.org/10.5755/j01.ms.23.3.16320

Received 30 September 2016; accepted 29 January 2017

\begin{abstract}
Multilayer colloidal crystal has been prepared by the layer-by-layer deposition of silica microspheres on a glass slide. Each layer is a slab consisting of a fcc close-packed colloidal arrays. By properly choosing the sizes of spheres, the whole spectral feature of multilayer colloidal crystal can be tuned. Here, we engineered a multilayer superlattice structure with an effective passband $(380 \mathrm{~nm})$ between two stop bands $(366 \mathrm{~nm}$ and $400 \mathrm{~nm})$. This gives a strong narrowing effect on emission spectrum $(378 \mathrm{~nm})$. With the stop bands at the shortwave and longwave edges of emission spectrum, the passband in the central wavelength region can be regarded as a strong decrease of suppression effect and enhancement of a narrow wavelength region of emission. The FWHM values of stop band modified emission spectrum were narrowed from $59 \mathrm{~nm}$ to $22 \mathrm{~nm}$. The spectral narrowing modification effect of suitably engineered colloidal crystals shows up their importance in potential application as optical filters and lasing devices.

Keywords: photonic crystal, stop band, passband, spectral narrowing, emission spectrum.
\end{abstract}

\section{INTRODUCTION}

Photonic crystals have received much attention in recent years for their potential of controlling light. Their dielectric periodicities give a forbidden region for photons, analogous to the band gap in electronic crystals, i.e., semiconductor crystals. The periodic structure gives rise to Bragg diffraction, which is associated with stop bands for propagation in certain directions, similar to the bandgap of electrons insemiconductors and represents that light with a range of energies, or wavelengths, is not allowed to propagate inside photonic crystals. In this case, photonic crystals offer a complete new mechanism for the control of light. Due to their special optical properties, photonic crystals have shown the potential applications as optical reflector, filters and switches, etc $[1-3]$. The numerous fabrication techniques of photonic crystals can roughly be categorized into two groups: top-down and bottom-up. Compared to top-down methods, which generally use lithography to obtain three-dimensional periodicities, bottom-up methods have been proven to be easier and more cost-effective. Typically, monodisperse colloidal spheres are assembled to arrays. A number of techniques have been developed, such as gravitational sedimentation $[4,5]$, deposition using a specially designed cell $[6,7]$, electrophoretic deposition $[8,9]$, vertical deposition using convective self-assembly [10], and Langmuir-Blodgett technique $[11,12]$, etc. At the first stage of investigation, scientists' attention is mainly focused on avoiding defects during the fabrication of colloidal photonic crystals, which can diminish the band gap effect of the whole crystal. With the enhancement of the fabrication techniques of three-

\footnotetext{
* Corresponding author. Tel.: +86-10-82543658; fax: +86-10-82543658

E-mail address: songkai@mail.ipc.ac.cn (K. Song)
}

dimensional photonic crystals, high-quality crystals can be obtained in large domains.

Like the heterostructures in semiconductors, some artificially introduced heterostructures in photonic crystals can also change their properties, including passband, stop band, and dispersion relation. In previous reports, different techniques were developed to introduce heterostructures into three-dimensional photonic crystals, including insertion a planar defect layer and combining slabs of spheres with different sizes [13-16].

The former involves the insertion of planar defect between two slabs of three-dimensional photonic crystals, while the defect layer has different lattice constant or refractive index comparing with the surrounding slab. Normally, for photonic crystals with this kind of heterostructures, there is a dip, also called passband, inside the stop band in transmission spectrum. The later method to fabricate heterostructures in photonic crystals is realized by layer-by-layer deposition of different opal slabs. The whole multilayer structure consists of several stacked slabs. Each slab is a close-packed array of colloidal spheres with different sizes or chemical composition [1719]. In recent years, we have explored new methods to insert a planar defect in photonic crystals, such as direct assembly at the air/water/substrate contract line [20].

With the prime aim of light localization [21], photonic crystal structures are also studied for the manipulation of the emission of fluorophores. The periodic dielectric properties can either provide feedback for improved lasing, or result in a forbidden region, the bandgap, where emission is suppressed and the fluorescence lifetime is enhanced. These effects have been studied by use of excited atoms, quantum dots, fluorescent molecules and, thermal radiation as point sources for photons [22-25]. Since the introduction of passband inside stop band from 
heterostructrues in photonic crystals, spectral narrowing of emission in colloidal photonic superlattices was reported, though the passband didn't give a clear observation in fluorescence emission spectrum [26,27]. What's more, due to the incomplete band gaps in colloidal crystals, the band gap effect on inside omnidirectional emission is weakened, while transmission spectrum is still the most appropriate to study the spectral features of passband and stop band [28].

In this report, we use multilayer colloidal crystal to give an overall structure with strong passband and stop band. By using outside fluorophore as the emission source, we clearly observed spectral narrowing effect of photonic crystals on fluorescence.

\section{EXPERIMENTAL}

\subsection{Materials}

All solvents and chemicals are of reagent quality and used without further purification except the water was of Milli-Q quality (Millipore, USA). Tetraethoxysilane (TEOS, $98 \%$, Acros) and $28 \%(\mathrm{w} / \mathrm{w})$ ammonium hydroxide was purchased from Alfa Aesar. Ethanol (a.p.) was obtained from Beijing Chemical works. Microslides $(75 \times 25 \times 1 \mathrm{~mm}$, Fisher) are cut into four smaller pieces with the size of $19 \times 25 \times 1 \mathrm{~mm}$. The fluorophore, recrystalized $\beta$-naphthalene methylformate was kindly provided by Prof. Li-Zhu Wu (Beijing, China).

\subsection{Preparation of monodisperse silica spheres}

Monodisperse silica spheres were synthesized following the Stöber-Fink-Bohn method through strict control of the reaction condition. In our case, reaction temperature was changed to adjust the size of obtained silica spheres, with fixed amounts of reagents (typically, $6 \mathrm{~mL}$ TEOS, $6.6 \mathrm{~mL} \mathrm{H} \mathrm{H}_{2} \mathrm{O}, 4.4 \mathrm{~mL} \mathrm{NH}_{3} \cdot \mathrm{H}_{2} \mathrm{O}$ and $100 \mathrm{~mL}$ $\mathrm{EtOH})$. Under vigorous stirring, TEOS was quickly added to the mixture of deionized water, $\mathrm{NH}_{3} \cdot \mathrm{H} 2 \mathrm{O}$ and ethanol in a $250 \mathrm{~mL}$ round bottom flask and react for at least $12 \mathrm{~h}$.

All the glass reaction vessels were extensively cleaned with hydrofluoric acid (2\%) and rinsed with water and ethanol prior to use. In order to study the band gap effect on emission of fluorophore, the proper size of silica spheres was chosen to match the stop bands with the emission of fluorophore. In a certain range, we found that the size of prepared silica spheres could be easily adjusted by slightly changing the reaction temperature. Also there was a good relation curve reaction temperature and the final size. With the decreasing of temperature, the spheres got larger. The two kinds of silica spheres we used in this study were prepared under $32{ }^{\circ} \mathrm{C}$ and $30^{\circ} \mathrm{C}$ separately, with the final sizes as $168 \mathrm{~nm}$ and $184 \mathrm{~nm}$.

\subsection{Fabrication of multilayer colloidal crystals}

After being purified by repeated centrifugation, the obtained monodisperse silica spheres were deposited onto the glass slides which were previously cleaned with piranha acid $(1 / 3(\mathrm{v} / \mathrm{v})$ sulfuric acid, 2/3(v/v) hydrogen peroxide as oxidant), to fabricate colloidal crystals in the method of convective self-assembly [10]. The final multilayer structure was obtained by repeated the deposition of colloidal crystal slab on a prepared one. Since the different sizes of silica spheres result in dimensional defects between two slabs of colloidal crystals, we deposited the slab of bigger spheres on the surface of the slab of smaller spheres. The thickness of each slab can be easily controlled by adjusting the concentration of colloidal suspension. In order to get the diffraction peaks of each slab of colloidal crystals with the similar intensities, the concentration of each colloidal suspension was carefully controlled.

\subsection{Characterization}

Spheres and the superlattice of colloidal crystal were observed by scanning electron microscopy (SEM, S-4300, Hitachi, Japan). Transmission spectra were recorded by UV-vis spectrometer (Lambda 35, Perkin Elmer), and taken along the [111] crystallographic axis which is normal to the direction of the glass slide.

To investigate the effect of stop band/pass band on the emission (spectral narrowing), $\beta$-naphthalene methyl formate has been chosen as the fluorophore. It's a solution in mixed solvents (the volume ratio of ethanol and water is 1:9) gives emission in the wavelength range between $335 \mathrm{~nm}-450 \mathrm{~nm}$, overlapping the stop band of our fabricated photonic crystals.

Emission spectra were measured by an F-4500 fluorescence spectrophotometer (Hitachi, Japan). We excited the dye with a beam of $280 \mathrm{~nm}$. The experimental setup is shown in Fig. 1.

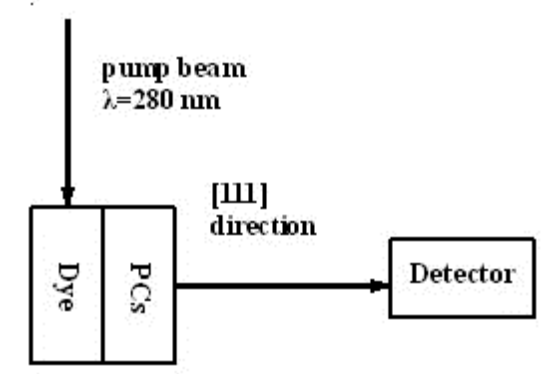

Fig. 1. Scheme of the optical setup. Using light of wavelength $280 \mathrm{~nm}$ as the excitation beam, the emitted light was detected under $90^{\circ}$ direction by spectrometer. The slide with colloidal crystal was carefully attached on the cell supporter, with the slab face to the spectrometer. Emitted light is collected normal and detected normal to the glass slide, of course, also to the (111) lattice plane of colloidal crystal

A slab of colloidal crystal on glass slide was placed between the detector and the quartz cell containing fluorophore solution. The glass slide was attached on the cell supporter, and perpendicular to the detecting direction. In this case, we can make sure that all the detected emitted light pass through the colloidal crystal and experience the strongest stop band/passband effects.

\section{RESULTS AND DISCUSSION}

\subsection{Morphology of photonic crystals and photonic superlattices}

Fig. 2 shows the typical scanning electron microscopy (SEM) top-view images of the photonic crystals films 
mentioned above and a cross-sectional micrograph of photonic crystal superlattice. These top-view images clearly indicate that colloidal spheres are in a FCC arrangement with the close-packed (111) plane oriented parallel to the substrates, with each sphere touching six others in one layer. The close-packed arrangement could extend over a large area (several square centimeters) [28]. The diameters of two kinds of silica spheres are A: $168 \mathrm{~nm}$ and B: $184 \mathrm{~nm}$. Their corresponding single crystals gave stop bands at the wavelength of $366 \mathrm{~nm}$ and $400 \mathrm{~nm}$, respectively.

Photonic superlattices were made by successive deposition of photonic crystal slabs composed of colloidal particles of the different sizes A and B. The slabs were deposited out of an ethanolic suspension of approximately 0.4 vol \%. From the cross-sectional views (Fig. 2 c), it can be observed that the colloidal superlattice exhibit a highly ordered structure. We deposited the slab of smaller spheres firstly on glass slide, in order to give upper slab with bigger spheres a relative flat bottom. And in Fig. 2, with the crystals of bigger spheres (diameter $184 \mathrm{~nm}$ ) as the top slab which grow on the surface of the bottom slab of smaller ones (diameter $168 \mathrm{~nm}$ ), the interface between the compositional colloidal crystal films was flat. The total thickness of the photonic crystal is about $3 \mu \mathrm{m}$ and consists of 20 layers of colloidal spheres.

The multilayer photonic superlattice exhibits dual photonic band gaps resulting from the linear combination of stop bands of the individual stacks (A at $366 \mathrm{~nm}$ and B at $400 \mathrm{~nm}$, respectively). Since the FWHM (full width at half maximum) of photonic stop band is proportional to the wavelength of stop band, with certain quality, here, we chose smaller silica spheres as building blocks to give sharper stop bands, and sequentially, deeper passband.

To study the effect of the stop band/passband on the emission properties (spectral narrowing) of fluorophore, different photonic crystal samples were made. Sample 1 is single slab photonic crystal of A; sample 2 is single slab photonic crystal of $\mathrm{B}$; and sample 3 is $\mathrm{AB}$ photonic crystal superlattice. Their extinction spectra are shown in Fig. 3.

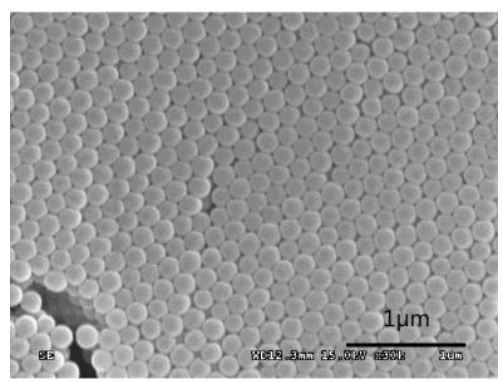

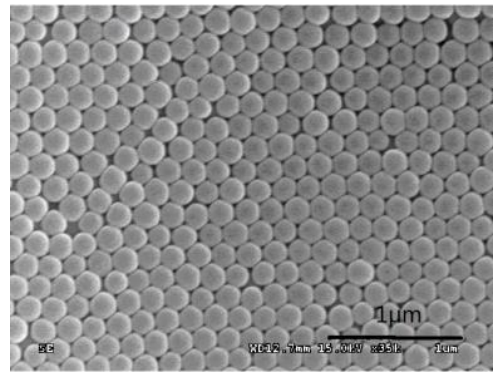

b

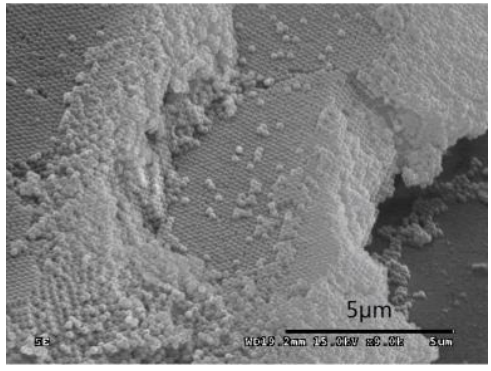

Fig. 2. SEM top-view images of silica colloid spheres with average diameter: $a-168 \mathrm{~nm} ; \mathrm{b}-184 \mathrm{~nm}$; $\mathrm{c}$-cross-section views of photonic superlattices of silica colloidal spheres with two different diameters (about 20 layers)

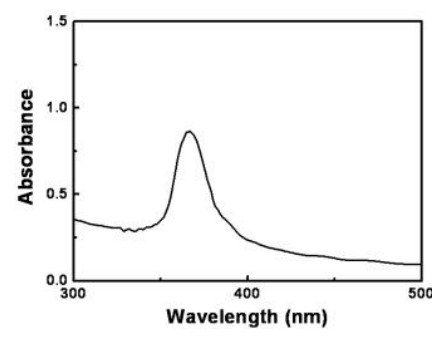

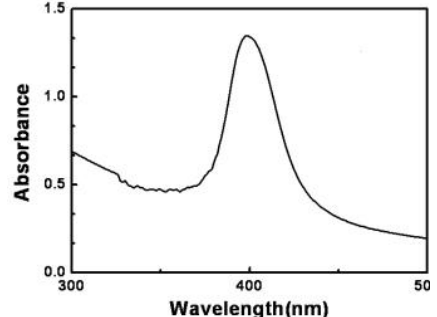

b

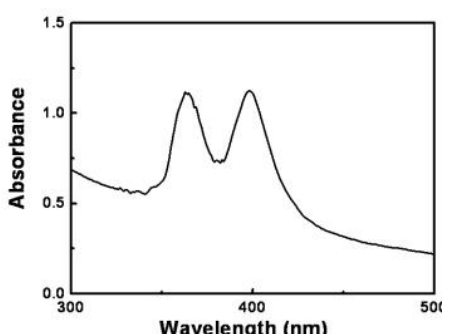

c

Fig. 3. Optical reflection spectrum of photonic: $\mathrm{a}$-crystal sample 1; b-sample 2; $\mathrm{c}-\mathrm{AB}$ superlattice sample 3

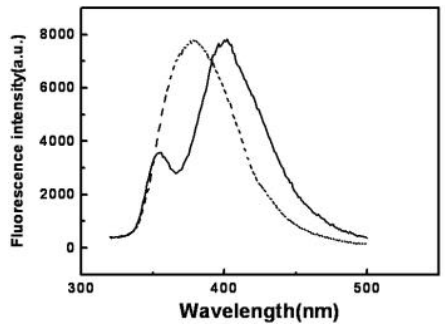

a

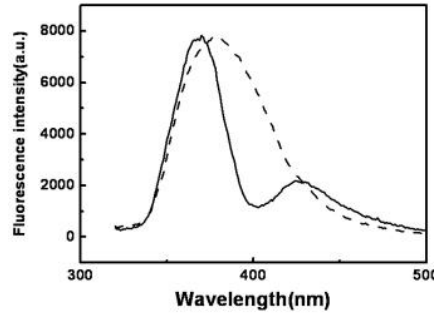

b

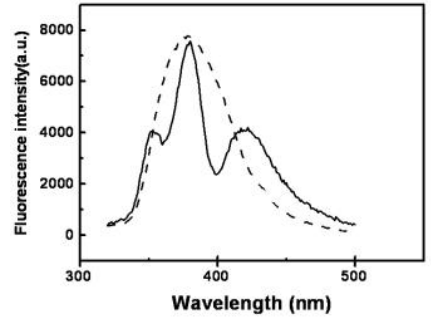

$\mathrm{C}$

Fig. 4. Modification of the fluorescence of dye by: a-sample 1; b-sample 2; c-sample 3; modified spontaneous emission spectrum of dye in photonic crystal (solid line), reference emission spectrum of dye in solution without stop band effect (dash line) 


\subsection{Spectral narrowing of emission}

To clearly observe the modification effect of photonic crystal on spontaneous emission, light sources must be placed in which strongly interacts with light. However, in most of the previous references, light sources such as dyes and lanthanide ions were introduced into photonic crystals just by simple infiltration or homogeneous embedding. In a previous report, it was found that when fluorophore was embedded inside photonic crystal, the stop band in the transmission spectrum was much deeper than the stop band in the emission spectrum, because of the omnidirectional nature of the emission and the incomplete band gap of silica photonic crystal [29].To study spectral features of passband and stop band in emission spectrum, in our case, we put fluorophore outside the photonic crystal, which can show strongest effect on the light emission in perpendicular direction.

To analyze the modification effect of photonic crystal on spontaneous emission spectra, the stop band and the passband were tuned by proper choice of sphere sizes and superlattices of colloidal crystals. $\beta$-Naphthalene methylformate was chosen as the fluorophore. In proper solvent, it gave spontaneous emission which overlapped the stop band region of our fabricated colloidal crystals.

We obtained fluorescence spectra of dye in different photonic crystal samples to analyze the relative influence of stop band and passband on emission. Comparisons of these modified spontaneous emission spectra are shown in Fig. 3 and Fig. 4 for sample $1-3$.

Fig. $4 \mathrm{a}$ and $\mathrm{b}$ show the fluorescence spectra of $\beta$ naphthalene methylformate dye in sample 1 and 2. For comparison, the corresponding transmission spectra are shown in Fig. $3 \mathrm{a}$ and b. The dips in emission spectra due to suppressing effect can be clearly observed, and they are in positions corresponding to the stop bands in transmission spectra. For each single-slab photonic crystal with the stop band locating at the edge of emission (sample 1 and sample 2), with the suppressing effect on emission, the spectral narrowing effects are also observed, However, simultaneously, suppression also results in a shift of emission peak.

In order to get stronger narrowing effect, we studied the modification effect of sample 3 (with multilayer structure) on emission spectrum. Between the linearly combined two stop bands, there is a dip, which is also called passband. Comparing with the passband caused by defect in photonic crystals reported previously [14-19], the dip in Fig. $4 \mathrm{c}$ is much deeper. Also, the combined stop band allows the complete overlapping of emission spectrum. In Fig. 3 c, we can see that two single stop bands are located at the shortwave edge and the longwave edge of the emission spectrum. Simultaneously, the passband of superlattice allows a better narrowing effect on the central wavelength region of emission.

For comparison, the FWHM values of each stop band modified emission spectrum were given in Table 1 . The data also support that engineered multilayer colloidal crystal can give a strong spectral narrowing effect on emission.

We also prepared another sample, which is a physical combination of two single slabs of colloidal crystals, by spectral feature of sample 3. That means that the second deposition of the slab of bigger silica spheres on the surface of the slab of smaller ones don't change much of the superlattice structure.

Table 1. Comparison of spectral features of modified emission spectra from different photonic crystal structures

\begin{tabular}{|c|c|c|c|}
\hline Crystal samples & $\begin{array}{c}\text { Stop band, } \\
\mathrm{nm}\end{array}$ & $\begin{array}{c}\text { Emission } \\
\text { maximum, nm }\end{array}$ & $\begin{array}{c}\text { FWHM total } \\
\text { stop band, nm }\end{array}$ \\
\hline- & - & 378 & 59 \\
\hline A & 366 & 402 & 49 \\
\hline B & 400 & 370 & 32 \\
\hline AB superlattice & 366,400 & 380 & 22 \\
\hline
\end{tabular}

\section{CONCLUSIONS}

Multilayer colloidal crystal has been fabricated by the layer-by-layer deposition of single slabs of silica microspheres. By changing the size of silica microspheres, the stop band of each slab of photonic crystal can be tuned. The spectral feature of the whole multilayer superlattice is a linear combination of stop bands of each single slab. In properly engineered multilayer superlattice, deep passband locates between two stop bands.

For emission outside photonic crystal, in this report, all the detected emitted light has strong interaction with (111) crystalline planes of colloidal crystal. We engineered $\mathrm{AB}$ photonic crystal superlattice by silica colloidal particles of different sizes A $(168 \mathrm{~nm})$ and B $(184 \mathrm{~nm})$. With the stop bands (A:366 nm and B:400 nm) at the shortwave and longwave edges of emission spectrum $(378 \mathrm{~nm})$. In this way, we observed a very strong and clear passband in emission spectrum, which is corresponding to the dip between two stop bands in the transmission spectrum. This reduction of suppression results in a spectral narrowing in emission spectrum. The FWHM values of stop band modified emission spectrum were narrowed from $59 \mathrm{~nm}$ to $22 \mathrm{~nm}$. In another word, it is a kind of enhancement of emission in a certain narrow wavelength region. It is believed that this experiment can be appreciated by considering engineered colloidal crystal with superlattices and heterostructures as a powerful tool for optical filter and lasing.

\section{Acknowledgements}

We acknowledge the financial support from the National Natural Science Foundation of China (21303173 and U1430128).

\section{REFERENCES}

1. Kamenetzky, E., Magliocco, L., Panzer, H. Structure of Solidified Colloidal Array Laser Filters Studied by Cryogenic Transmission Electron Science 263 (5144) 1994: pp. 207-210.

2. Dudok, T.H., Nastishin, Y.A. Optically Pumped Mirrorless Lasing. A Review: Part II. Lasing in Photonic Crystals and Microcavities Ukrainian Journal of Physical Optics 15 (2) 2014: pp. 47-67.

https://doi.org/10.3116/16091833/15/2/47/204

3. Han, S.G., Lim, J., Shin, J., Lee, S.M., Park, T., Yoon, J., Woo, K., Lee, H., Lee, W. Optically Pumped Distributed Feedback Dye Lasing with Slide-Coated $\mathrm{TiO}_{2}$ 
Inverse-Opal Slab as Bragg Reflector Optics Letters 39 (16) 2014: pp. $4743-4746$.

4. Wijnhoven, J., Vos, W.L. Preparation of Photonic Crystals Made of Air Spheres in Titania Science 281 (5378) 1998: pp. 802-804.

5. Yetisen, A.K., Naydenova, I., da Cruz Vasconcellos, F., Blyth, J., Lowe, C.R. Three-Dimensional AnalyteSensitive Nanostructures and Their Applications Chemical Reviews 114 (20) 2014: pp. 10654-10696.

6. Gates, B., Qin, D., Xia, Y. Assembly of Nanoparticles into Opaline Structures over Large Areas Advance Materials 11 (6) 1999: pp. 466-469.

7. Kang, K., Huang, S., Feng, Y., Dong, L., Zhu, L. Fabrication of Colloidal Photonic Crystals by a Micro-cell Based on Al Thin Film Acta Chimica Sinica 66 (14) 2008: pp. $1615-1619$.

8. Holgado, M., Garcia-Satamaria, F., Blanco, A., Ibistate, M., Cintas, A., Miguea, H., Serna, C.J., Molpecers, C., Requena, J., Mifsud, A., Meseguer, F., Lopez, C. Electrophoretic Deposition to Control Artificial Opal Growth Langmuir 15 (14) 1999: pp. 4701-4704.

9. Zhang, J.P., Yu, L.P. Preparation of Three-Dimensional Ordered Macroporous $\mathrm{Cu}_{2} \mathrm{O}$ Film through Photonic Crystal Template-Assisted Electrodeposition Method Journal of Materials Science: Materials in Electronics $25(12)$ 2014: pp. 5646-5651. https://doi.org/10.1007/s10854-014-2354-8

10. Jiang, P., Bertone, J.F., Hwang, K.S., Colvin, V.L. Single-Crystal Colloidal Multilayers of Controlled Thickness Chemistry of Materials $11(8)$ 1999: pp. 2132-2140.

11. Reculusa, S., Ravaine, S. Synthesis of Colloidal Crystals of Controllable Thickness through the Langmuir-Blodgett Technique Chemistry of Materials 15(2) 2003: pp. 598-605. https://doi.org/10.1021/cm021242w

12. Bardosova, M., Pemble, M.E., $\quad$ Povey, I.M., Tredgold, R.H. The Langmuir-Blodgett Approach to Making Colloidal Photonic Crystals from Silica Spheres Advance Materials 22 (29) 2010: pp. 3104-3124.

13. Wostyn, K., Zhao, Y., Schaetzen, G., de Hellemans, L., Matsuda, N., Clays, K., Persoons, A. Insertion of a TwoDimensional Cavity into a Self-Assembled Colloidal Crystal Langmuir 19 (10) 2003: pp. 4465-4468.

14. Zhao, Y., Wostyn, K., Schaetzen, G. de Clays, K., Hellemans, L., $\quad$ Persoons, A., $\quad$ Szekeres, M., Schoonheydt, R.A. The Fabrication of Photonic Band Gap Materials with A Two-Dimensional Defect Applied Physics Letters 82 2003: pp. 3764-3767. https://doi.org/10.1063/1.1578713

15. Hong, P.N., Benalloul, P., Coolen, L., Maître, A., de Schwo, C.J. A Sputtered-Silica Defect Layer between Two Artificial Silica Opals: An Efficient Way to Engineer Well-Ordered Sandwich Structures Journal of Materials Chemistry C 1 2013: pp. 5381-5386. https://doi.org/10.1039/c3tc30697f

16. Massé, P., Reculusa, S., Clays, K., Ravaine, S. Tailoring Planar Defect in Three-Dimensional Colloidal Crystals Chemical Physics Letters 422 (1-3) 2006: pp. 251-255.
17. Jiang, P., Ostojic, G.N., Mittleman, R.M., Colvin, V.L. The Fabrication and Bandgap Engineering of Photonic Multilayers Advance Materials 13 (6) 2001: pp. 389-393.

18. Egen, M., Voss, R., Griesebock, B., Zentel, R. Heterostructures of Polymer Photonic Crystal Films Chemistry of Materials 15 (20) 2003: pp. 3786-3792.

19. Ding, T., Song, K., Clays, K., Tung, C.H. Bottom-Up Photonic Crystal Approach with Top-Down Defect and Heterostructure Fine-Tuning Langmuir $26(6)$ 2010: pp. $4535-4539$.

20. Zhong, K., $\quad$ Demeyer, P.J., Z Zhou, X., Kruglova, O., Verellen, N., Moshchalkov, V.V., Song, K., Clays, K. A Facile Way to Introduce Planar Defects into Colloidal Photonic Crystals for Pronounced Passbands Journal of Materials Chemistry C 2014: pp.8829-8836.

21. John, S. Strong Localization of Photons in Certain Disordered Dielectric Superlattices Physical Review Letters 58 1987: pp. 2486-2489.

22. Gaponenko, S.V., Bogomolov, V.N., $\quad$ Petrov, E.P., Kapitonov, A.M., Yarotsky, D.A., Kalosha, I.I., Eychmueller, A.A., Rogach, A.L., McGilp, J., Woggon, U., Gindele, F.J. Spontaneous Emission of Dye Molecules, Semiconductor Nanocrystals, and Rare-Earth Ions in Opal-Based Photonic Crystals Journal of Lightwave Technology 17 (11) 1999: pp. 2128-2137. https://doi.org/10.1109/50.803003

23. Petrov, E.P., Bogomolov, V.N., Kalosha, I.I., Gaponenko, S.V. Spontaneous Emission of Organic Molecules Embedded in a Photonic Crystal Phyical Review letters 81 1998: pp. 77-82.

24. Li, H., Wang, J., Lin, H., Xu, L., Xu, W., Wang, R., Song, Y., Zhu, D. Amplification of Fluorescent Contrast by Photonic Crystals in Optical Storage Advance Materials 22 (11) 2010: pp. 1237-1241.

25. Lodahl, P., Driel, A.F., van Nikolaev, I.S., Irman, A., Overgaag, K., $\quad$ Vanmaekelbergh, D., $\quad$ Vos, W.L. Controlling the Dynamics of Spontaneous Emission from Quantum Dots by Photonic Crystals Nature 430 2004: pp. 654-657.

26. Baert, K., Song, K., $\quad$ Vallée, R., $\quad$ Auweraer, M., Van der Clays, K. Spectral Narrowing of Emission in SelfAssembled Colloidal Photonic Superlattices Journal of Applied Physics 100 2006: pp. 123112-123116. https://doi.org/10.1063/1.2402029

27. Li, H., Wang, J., Liu, F., Song, Y., Wang, R. Fluorescence Enhancement by Heterostructure Colloidal Photonic Crystals with Dual Stopbands Journal of Colloid and Interface Science 356 (1) 2011: pp. 63-68.

28. Song, K., Vallée, R., Van der Auweraer, M., Clays, K. Fluorophores-Modified Silica Sphere as Emission Probe in Photonic Crystals Chemical Physics Letters $421(1-3)$ 2006: pp. $1-4$.

29. Wang, J., Wen, Y., Ge, H., Sun, Z., Zheng, Y., Song, Y., Jiang, L. Simple Fabrication of Full Color Colloidal Crystal Films with Tough Mechanical Strength Macromolecular Chemistry and Physics 207 (6) 2006: pp. 596-604. 